\title{
Role of the Cardiophrenic Lymph Node Status After Neoadjuvant Chemotherapy in Primary Advanced Ovarian Cancer
}

\author{
VERONICA LUENGAS-WUERZINGER ${ }^{1}$, FRIEDERIKE RAWERT ${ }^{1}$, SABRINA CLAßEN-VON SPEE ${ }^{1}$, \\ SAHER BARANSI ${ }^{1}$, ESTHER SCHULER ${ }^{1}$, KATHARINA CARRIZO $^{1}$, PETER MALLMANN $^{2}$ and BJOERN LAMPE ${ }^{1}$ \\ ${ }^{1}$ Florence-Nightingale Hospital, Department of Gynaecology and Obstetrics, Düsseldorf, Germany; \\ ${ }^{2}$ University Hospital of Cologne, Department of Obstetrics and Gynaecology, Cologne, Germany
}

\begin{abstract}
Background/Aim: This study investigated the cardiophrenic lymph node (CPLN) status before and after neoadjuvant chemotherapy (NACT), as its presence seems to have a rather prognostic significance in patients with advanced ovarian cancer. Patients and Methods: The baseline computed tomography scans of 66 patients with advanced ovarian cancer primary treated with NACT between March 2015 and June 2020 were reviewed. A CPLN enlargement was defined as $\geq 5 \mathrm{~mm}$. Results: $44 \%(n=29)$ of the patients had enlarged CPLNs; $10.7 \%(n=3)$ showed a complete response, $71.4 \%(n=20)$ a partial response, and $17.9 \%(n=5)$ a stable disease after NACT. There was no significant difference between the response to NACT measured according to the status of CPLN compared to other biomarkers in the CPLN group. Conclusion: Patients with CPLN enlargement have a tendency to an impaired prognosis. The response of CPLN to NACT was comparable to the response of established biomarkers, adding a monitoring function to the CPLN.
\end{abstract}

Ovarian cancer is the second most lethal gynaecologic malignancy in Germany as it is mostly diagnosed at an advanced stage (76\% stage III/IV), with a 5-year survival rate of $43 \%$ (1). Primary cytoreductive surgery followed by chemotherapy has been the standard treatment for patients

This article is freely accessible online.

Correspondence to: Verónica Luengas-Würzinger, FlorenceNightingale Hospital, Department of Gynaecology and Obstetrics, Kreuzbergstr. 79, 40489 Düsseldorf, Germany. Tel: +49 2114092519, Fax: +49 2114092254, e-mail: luengaswuerzinger@kaiserswerther-diakonie.de.

Key Words: Ovarian neoplasms, neoadjuvant therapy, lymphatic metastasis, computed tomography. with advanced ovarian cancer over the recent decades. An alternative approach of interval debulking surgery following neoadjuvant chemotherapy (NACT) has been investigated in different trials (2-5).

There are well-established prognostic factors for ovarian cancer: the initial International Federation of Gynaecology and Obstetrics (FIGO) stage, a macroscopic complete resection after surgery, histological type and grading, general state of health, and patient's age. The once regularly performed systematic lymphadenectomy at the time of surgery has lost significance since the Lymphadenectomy in Ovarian Neoplasms (LION) trial showed no survival benefits in patients with advanced ovarian cancer and radiologic and clinical unsuspicious retroperitoneal nodes (6). In contrast, the role of the frequently observed enlarged cardiophrenic lymph nodes (CPLNs) has only scarcely been investigated.

The definition of pathologically enlarged CPLN has not been standardized. According to the Response Evaluation Criteria in Solid Tumours (RECIST) guidelines (version 1.1) (7), lymph nodes, regardless of their location, are pathologically enlarged when their short-axis is $>10 \mathrm{~mm}$ (8). However, diverse studies describe a negative impact in the prognosis of patients with ovarian cancer showing CPLNs with a short-axis of $>5 \mathrm{~mm}$ (9-12). Hence, we defined a CPLN enlargement according to the ESUR guidelines with a cut-off $>5 \mathrm{~mm}$ short axis dimension (13).

Anatomically, CPLNs are located above the abdominal cavity between the mediastinum, the heart base, diaphragm and the chest wall, defining a FIGO stage of IVB in ovarian cancer if they are enlarged or histologically positive. Still, previous studies have shown that the survival of ovarian cancer patients relies more on a complete intraabdominal tumour resection, even if the CPLN remain surgically unremoved $(9,14)$. Therefore, according to existing literature, the presence of enlarged CPLN seems to have a rather prognostic than therapeutic significance. 

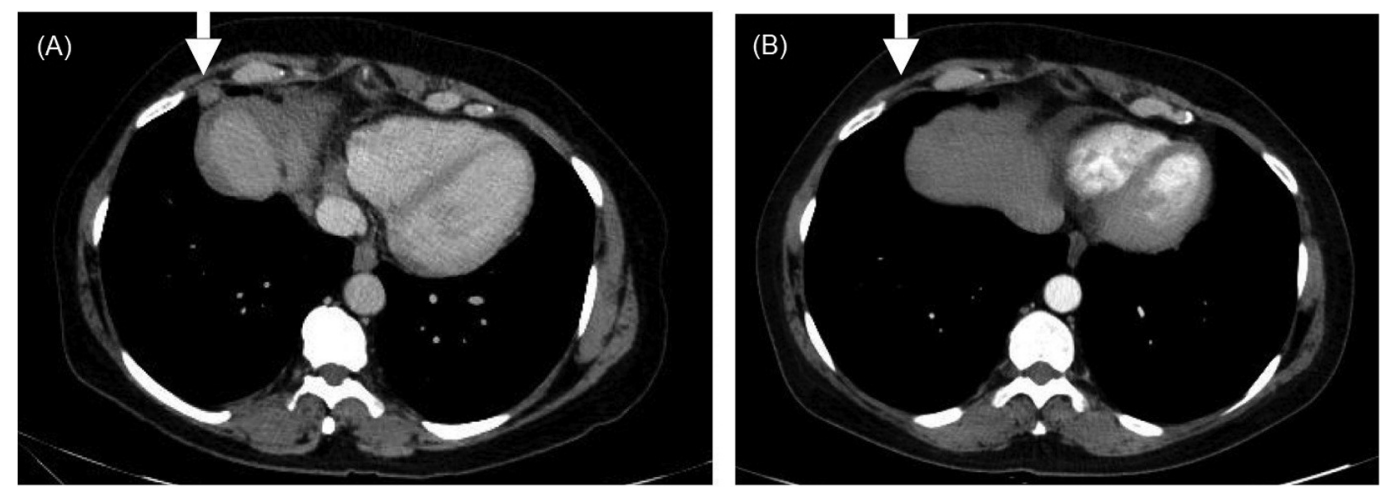

Figure 1. Example of a cardiophrenic lymph node with a short axis of $7 \mathrm{~mm}$, before $(A)$ and after (B) neoadjuvant chemotherapy.

The purpose of this retrospective report was to analyse the role of the CPLN status before and after NACT (Figure 1) and to compare its value and response with other clinical and radiological parameters such as the presence of ascites, radiologic enlarged pelvic and para-aortic lymph nodes (PPLNs), CA 125, HE4, the place of recurrence and the Sugarbaker Peritoneal Cancer Index (PCI). The PCI-score was established to objectify the extend of peritoneal carcinomatosis and to predict operability in general surgery (15). It is a useful extension of the existing classification systems for the treatment of OCs and is being used as a parameter for the assessment of resectability in our hospital (16).

\section{Patients and Methods}

The study was approved by the appropriate Institutional Review Board (IRB), which waived the requirement for written informed consent.

Between 03/2015 and 06/2020 a total of 66 patients with primary diagnosed FIGO (2014) stage IIIb-IVb ovarian, tubal, and peritoneal carcinoma were treated with NACT as a primary therapy in the Florence Nightingale Hospital in Düsseldorf (e.g., in case of inoperability, patients wish, patients that had already received NACT and were referred to our hospital for surgery etc.). All patients underwent staging examinations at the time of diagnosis, including CT scans of the thorax and abdomen, lab works, as well as a diagnostic laparoscopy for histological confirmation of the diagnosis and evaluation of operability. The baseline CT scans of these 66 patients were retrospectively reviewed by our radiologist. To avoid selection bias, the radiologist blindly and randomly analysed the CT scans of all patients without knowing the time of the study or the patient's name. According to the ESUR guidelines, we defined a pathological CPLN enlargement at a cut-off of $>5 \mathrm{~mm}$ short axis dimension (13). The analysis was performed using patient files, surgical records, radiological records, and intraoperative imaging documentation.

The patients were radiologically classified into a group with $(n=29)$ and without $(n=37)$ enlarged CPLNs at the time of diagnosis. These two groups were compared regarding clinicopathological parameters like the presence of radiologic enlarged PPLNs, presence of ascites, CA 125, HE4 and the Sugarbaker PCI before and after NACT, as well as the time and place of recurrence.

These associations were evaluated using Mann-Whitney $U$ or Chi-square tests, defining a statistical significance as $p \leq 0.05$. The differences in survival were determined using the Kaplan-Meier method with log-rank test and Cox's proportional hazard models for uni- and multi-variate analyses.

Among the group with enlarged CPLNs, the response to chemotherapy of the different clinicopathological parameters was evaluated and compared using the linear regression, the binary logistic regression, and the Spearman's rank correlation.

All statistical analyses were performed using the IBM Statistical Product and Service Solutions (SPSS ${ }^{\circledR}$ ) Statistics software version 27 for Macintosh (IBM, Armonk, NY, USA).

\section{Results}

In a cohort of 66 patients with advanced ovarian cancer undergoing NACT, 44\% $(n=29)$ had radiologically enlarged CPLNs and $56 \%(n=37)$ had CPLN with a short-axis of $<5$ $\mathrm{mm}$ in the CT scan before starting the therapy. These two groups were analysed at the time of diagnosis for the amount of ascites, CA125 and HE4-levels, preoperative Sugarbaker PCI, and the presence of enlarged PPLNs. Additionally, the residual disease after surgery, the time and site of recurrence, and the progression-free survival (PFS) of these two groups were evaluated.

The median age at diagnosis was 65 years [interquartile range $(\mathrm{IQR})=57-75]$. We observed a recurrence in $59 \%$ $(n=39)$ of the patients and the median PFS was 14 months (IQR=7-20.5). Eighteen (27\%) patients died during a median time of follow up of 18 months (IQR=10-29.5).

The levels of CA 125 and HE4, the amount of ascites, the presence of intraabdominal retroperitoneal lymph nodes, and the preoperative Sugarbaker PCI were not significantly different between the two groups at the time of diagnosis (Table I).

Neither did the presence of enlarged CPLNs significantly correlate with a postoperative Sugarbaker PCI, nor did 
Table I. Patient characteristics $(n=66)$

\begin{tabular}{|c|c|c|c|c|c|}
\hline & \multicolumn{2}{|c|}{ CPLN short-axis $\geq 5 \mathrm{~mm}(\mathrm{n}=29)$} & \multicolumn{2}{|c|}{ CPLN short-axis $<5 \mathrm{~mm}(\mathrm{n}=37)$} & \multirow[b]{2}{*}{$p$-Value } \\
\hline & Median & $\mathrm{IQR}(\%)$ & Median & $\mathrm{IQR}(\%)$ & \\
\hline \multicolumn{6}{|l|}{ FIGO stage } \\
\hline IIIA/B & 9 & 31 & 9 & 24.3 & \\
\hline IIIC & 17 & 58.6 & 23 & 62.2 & \\
\hline IVA/B & 3 & 10.3 & 5 & 13.5 & \\
\hline \multicolumn{6}{|c|}{ Residual disease after surgery } \\
\hline Yes & 4 & 13.8 & 5 & 13.5 & \\
\hline No & 25 & 86.2 & 32 & 86.5 & 0.974 \\
\hline \multicolumn{6}{|l|}{ Recurrence } \\
\hline Yes & 19 & 65.5 & 20 & 54.1 & \\
\hline No & 10 & 34.5 & 17 & 45.9 & 0.347 \\
\hline \multicolumn{6}{|c|}{ Place of recurrence } \\
\hline Abdominal & 14 & 87.5 & 14 & 73.7 & \\
\hline Thoracal & 1 & 6.3 & 1 & 5.3 & \\
\hline Combined & 1 & 6.3 & 3 & 15.8 & 0.625 \\
\hline \multicolumn{6}{|l|}{ Enlarged PPLN } \\
\hline Yes & 8 & 27.6 & 6 & 16.2 & 0.262 \\
\hline No & 21 & 72.4 & 31 & 83.8 & \\
\hline \multicolumn{6}{|l|}{ Sugarbaker PCI } \\
\hline Before NACT & 18.1 & $8-30$ & 18.1 & $6-27.8$ & 0.872 \\
\hline After NACT & 8.07 & $0.5-13.5$ & 9.05 & $0-15.5$ & 0.733 \\
\hline \multicolumn{6}{|l|}{ CA125 (U/ml) } \\
\hline Before NACT & 868.8 & $258-1240$ & 1866 & $98.8-928.8$ & 0.342 \\
\hline After NACT & 128.2 & $12.9-102.8$ & 195.9 & $23.2-66.8$ & 0.146 \\
\hline \multicolumn{6}{|l|}{ Ascites $(0-3) *$} \\
\hline Before NACT & 1.66 & $1-3$ & 1.38 & $0.00-3.00$ & 0.341 \\
\hline After NACT & 0.19 & $0.00-0.00$ & 0.24 & $0.00-0.00$ & 0.888 \\
\hline \multicolumn{6}{|l|}{ HE4 } \\
\hline Before NACT & 690.37 & $152-815$ & 892.23 & $122-1125$ & 0.707 \\
\hline After NACT & 188.45 & $72.93-296.0$ & 126.13 & $58.6-128.0$ & 0.245 \\
\hline PFS & \multicolumn{2}{|c|}{$\mathrm{HR}=1.26$} & \multicolumn{2}{|c|}{$95 \% \mathrm{CI}=0.67-2.38$} & 0.476 \\
\hline OS & \multicolumn{2}{|c|}{$\mathrm{HR}=1.15$} & \multicolumn{2}{|c|}{$95 \% \mathrm{CI}=0.45-2.93$} & 0.768 \\
\hline
\end{tabular}

CPLN: Cardiophrenic lymph node; NACT: neoadjuvant chemotherapy; IQR: interquartile range; FIGO: International Federation of Gynaecology and Obstetrics; PPLN: pelvic and para-aortic lymph nodes; PCI: peritoneal cancer index; PFS: progression-free survival; OS: overall survival. *The amount of ascites was objectified as follows: $0=$ no ascites, $1=$ minimal ascites, $2=$ moderate ascites, $3=$ massive ascites.

operability (macroscopic tumour clearance after surgery), achieving a comparable tumour reduction in both groups after NACT.

Patients with enlarged CPLN had a higher probability of getting a recurrence than patients without CPLN $(\mathrm{HR}=1.26)$, however the difference was not statistically significant $(p=0.476)$ (Figure 2). The place of recurrence also did not significantly vary between the two groups.

Response to NACT measured by the CPLN in the group with pathologically enlarged CPLNs $(n=29)$ was evaluated. Of these patients, $10.7 \%(n=3)$ showed complete response, $71.4 \%(n=20)$ showed partial response and $17.9 \%(n=5)$ had stable disease according to RECIST guidelines (version 1.1) (7) after NACT. None of the patients had progressive disease in this patient group. One patient was ruled out in regard to the response to NACT, as she did not undertake a control CT scan after chemotherapy.

There was no significant difference between the response to NACT as measured by the CPLN and the response to chemotherapy measured by other factors like PPLN, the CA125-level, the HE4-level, the Sugarbaker PCI, and the amount of ascites.

The response to chemotherapy measured by the CPLN did not significantly influence operability (possibility to reach a macroscopic tumour clearance) $(p=0.726)$. However, the bigger the reduction in the CPLN after chemotherapy, the higher was the probability of getting a recurrence $(p=0.013)$ (Figure 3). 


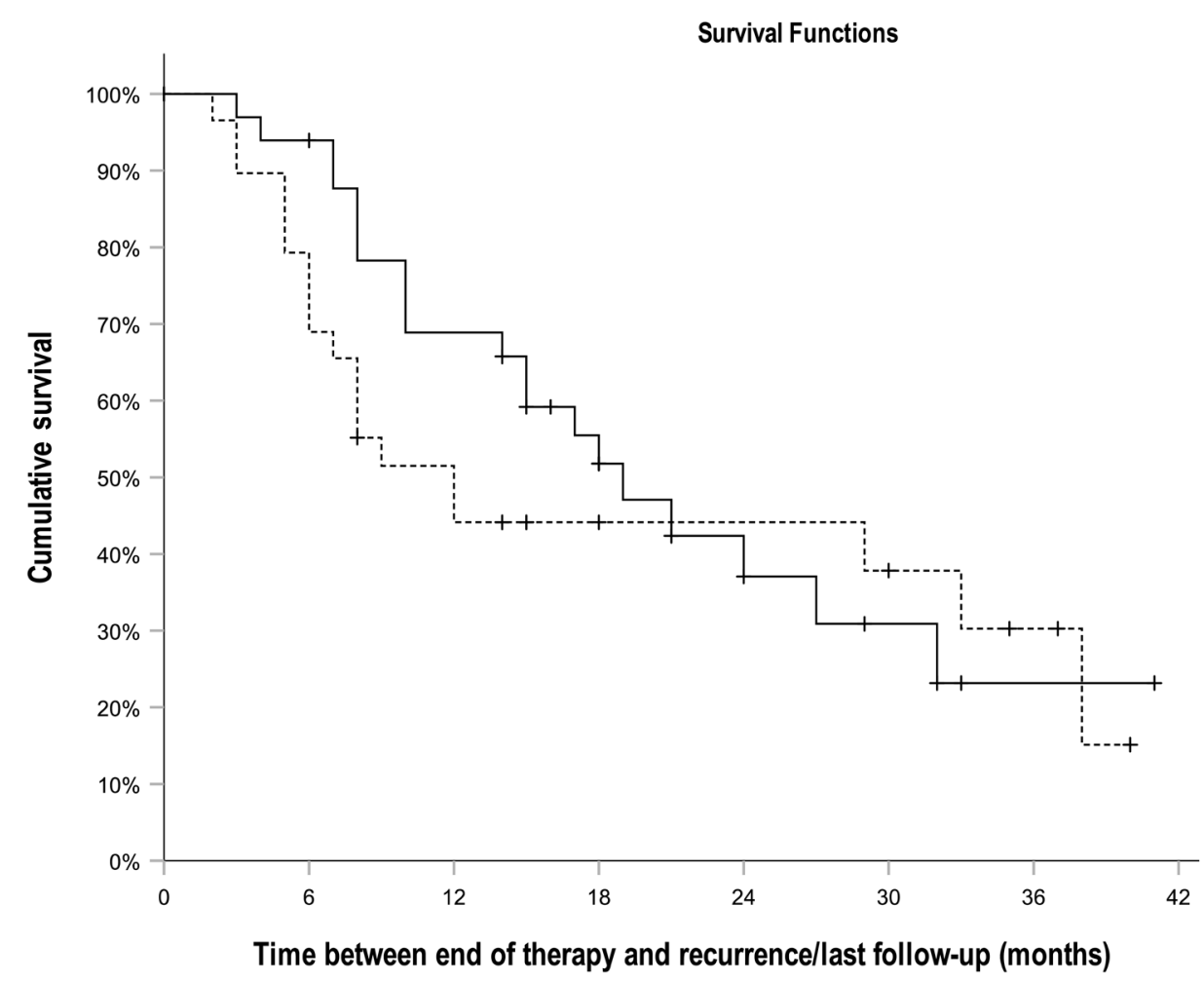

\section{Enlarged cardiophrenic lymph nodes (CPLNs) \\ $\neg$ No \\ $-n^{--}$YYes \\ + No-censored \\ + Yes-censored}

Figure 2. Progression-free survival in the cardiophrenic lymph node (CPLN) positive and negative group.

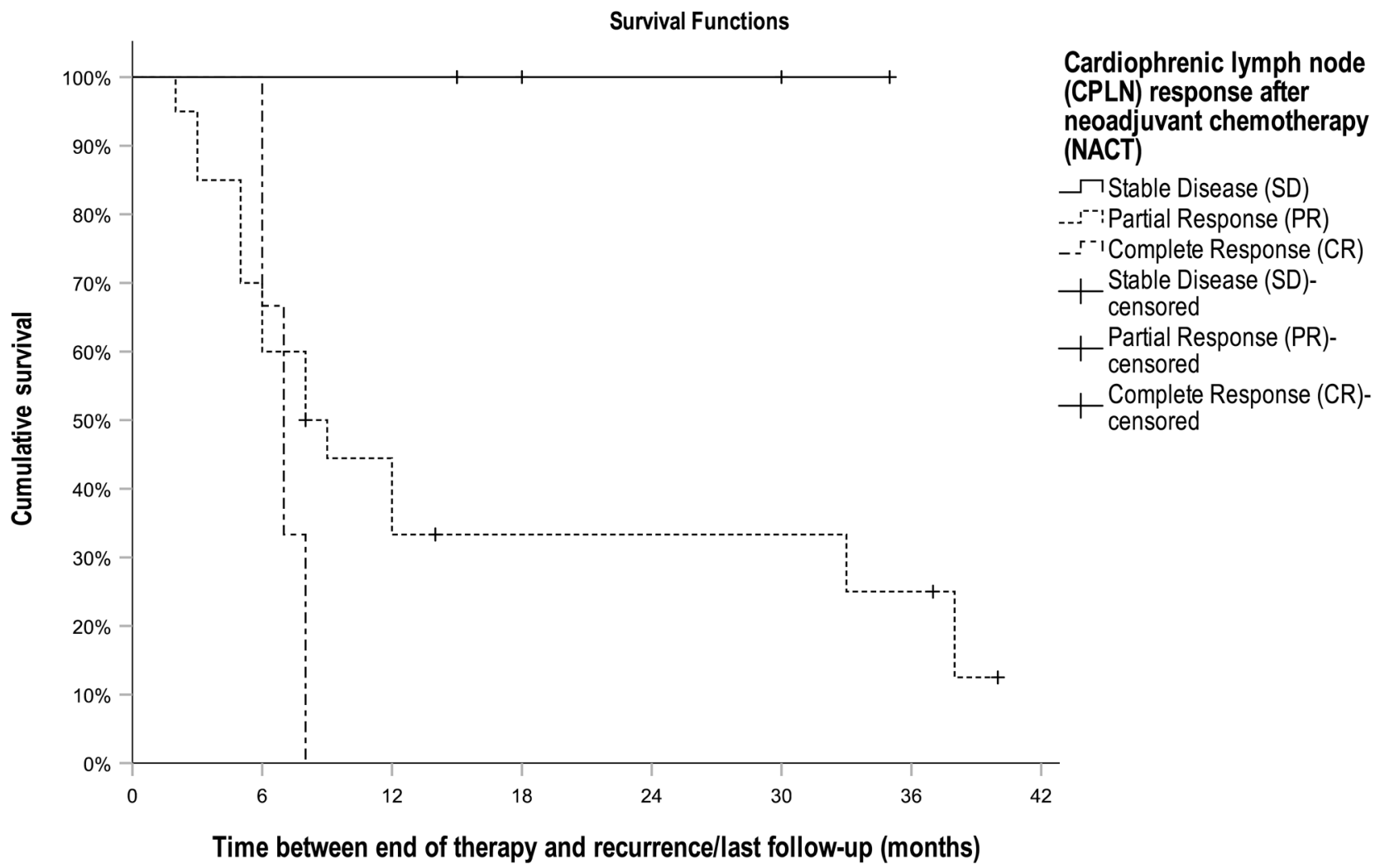

Figure 3. Cardiophrenic lymph node (CPLN) response after neoadjuvant chemotherapy. 


\section{Discussion}

Summary of main results. Our study showed that response to NACT can also be measured by the CPLN in advanced ovarian cancer. This response is comparable to the response measured by established biomarkers (e.g., CA125, HE4, the Sugarbaker PCI, and the amount of ascites).

With a cut-off value of $5 \mathrm{~mm}$ short axis to define a CPLN enlargement, $82 \%$ of our patients showed at least a partial response to NACT. The bigger the reduction, the higher was the probability of getting a recurrence in these patients, supporting the negative prognostic value of the CPLN. The analysed biomarkers at the time of diagnosis did not significantly vary between the group with enlarged CPLN and the group without.

Results in the context of published literature. In our study cohort, $44 \%$ of the patients had radiologic enlarged CPLNs (short axis $\geq 5 \mathrm{~mm}$ ). In the literature, the described CPLNdetection rates vary between $10.5 \%$ and $62 \%(8,9,17-19)$, depending on the short axis diameter threshold determined for the patient cohort. The higher the short-axis threshold, the lower the detection rate of potentially pathological CPLNs (20). This could be confirmed in our study cohort: if defining a CPLN enlargement at a short-axis of $\geq 7 \mathrm{~mm}$ or $\geq 10 \mathrm{~mm}$, the percentage of patients with radiologically enlarged CPLNs would have been reduced to $23 \%(n=15)$ and $6 \%(n=4)$, respectively. Therefore, we chose the ESUR guidelines defined radiologic cut-off of a CPLN short-axis of $\geq 5 \mathrm{~mm}$ (13), as there are also data describing histological detection rates up to $84 \%$ with this cut-off (9). This percentage correlates with the response to NACT in $82 \%$ of the patients with enlarged CPLNs in our study.

The removal of CPLNs was not routinely performed at the interval debulking surgery in our patient cohort, as its radiological presence was interpreted differently depending on the cut-off value the radiologist used at that time. If the presence of enlarged CPLN would have been considered with a cut-off of $\geq 5 \mathrm{~mm}$, it would have reflected as extraabdominal disease involvement causing a stage shift from FIGO III to FIGO IVb in up to $39 \%(n=26)$ of our patient cohort. Despite this possible shift, the removal of CPLNs would not have changed the therapy after surgery.

Even if the enlarged CPLNs at the time of diagnosis were not histologically confirmed in the majority of our patients, $82.1 \%$ showed at least a partial response to NACT according to RECIST-guidelines (version 1.1). This percentage is comparable to the percentage of histologically confirmed CPLNs described in the literature, lying between $61 \%$ and $90 \%(9,10,17,21,22)$. In line with this, the presence of CPLN metastases in around $82.1 \%$ of the patients with enlarged CPLN in our patient cohort by means of their response to NACT can be assumed (23), even if they were not histologically confirmed. The significant higher probability of getting a recurrence in patients with a higher reduction in the CPLN after chemotherapy also supports this hypothesis.

Research has shown that patients with radiologically enlarged CPLNs have a worse overall survival (9, 18-20, 24, 25), strengthening its clinical importance. Our data also corroborated a tendency to an impaired prognosis in patients with radiologically enlarged CPLNs, though the time of follow up was too short to be statistically significant. On the other hand, Prader et al. revealed that the survival of ovarian cancer patients depends more on a complete intraabdominal tumour resection, even if the CPLN remain surgically unremoved (9). Hence, the intraabdominal tumour spreading in ovarian cancer seems to have a greater impact than the lymphatic spread. This hypothesis is also confirmed by the findings of the LION-trial, were systematic pelvic and paraaortic lymphadenectomy in patients with advanced ovarian cancer was not associated with longer overall survival or PFS, although hidden lymph node metastases were detected in $55.7 \%$ of the patients (6). In our study cohort, $90 \%$ of the patients could be operated after NACT without residual disease regardless of the presence of CPLNs. During the follow-up time, there was neither a significant difference in the probability of getting a recurrence, nor did the site of recurrence vary between the patients with radiologic enlarged CPLNs and the ones with CPLNs with a short-axis of $<5 \mathrm{~mm}$.

Our data demonstrate no significant correlation between enlarged CPLNs and the analysed clinical parameters at the time of diagnosis (Table I). Especially the association between the presence of enlarged CPLN and PPLN could not be proven, which also supports the importance of the intraabdominal over the lymphatic spread in ovarian cancer. Pader et al. showed a weaker association between PPLN positivity and CPLN compared to the association of upper abdominal/diaphragmatic carcinomatosis. In addition, we found no significant difference in the response to NACT as measured by the CPLN compared to the response of other biomarkers in the same group of patients. This suggests that the presence of CPLNs itself could have not only a prognostic, but also a monitoring function regarding chemotherapy, which is comparable to the function of other already established biomarkers.

Strengths and weaknesses. So far, there are no previous studies evaluating the CPLN status before and after NACT in advanced ovarian cancer, which gives a new point of view of its implications regarding its monitoring function. By means of the response to chemotherapy, it can be indirectly inferred as a tumour involvement even if the CPLN status is not histologically confirmed.

Our study though has some limitations. Radiologically enlarged CPLNs were not histologically confirmed, as the study relied only on their size. Therefore, we can only make indirect 
conclusions regarding their response to chemotherapy. As a pilot study, the study cohort was quite small, and thus our statistical analysis was often not significant. In addition, we selected a patient group treated between 03/2015 and 06/2020, therefore the time used to analyse PFS was too short. The retrospective design of our study may have also caused a selection bias.

Implications for practice and future research. CPLN enlargement in ovarian cancer is not yet a well-established prognostic parameter, despite the fact that it has been shown to correlate with a worse overall survival. Identifying the CPLN status at the time of diagnosis would help to stage patients correctly into a FIGO IVb stage. For this, a general radiological cut-off value to describe the CPLN enlargement should be defined. In addition, the CPLN status seems to also have a monitoring value after chemotherapy.

Larger analysis including data of NACT-treated patients in other centres should be performed to fully evaluate the implication of the CPLN in advanced ovarian cancer.

\section{Conclusion}

Consistent with the literature, our data revealed a tendency to an impaired prognosis for patients with radiological CPLN enlargement $(9,18-20,26)$. Yet, this correlation was not significant in our patient cohort, probably due to the small number of patients and short follow-up time. Including the CPLNs into the FIGO classification for ovarian cancer would mean an extra abdominal tumour involvement, aggravating the stage of the disease at the time of diagnosis in many patients. For this, a general radiological cut-off threshold for the short axis should be defined. Our data showed that with a radiologic cut-off of $>5 \mathrm{~mm}$ for the CPLN, it is possible to infer tumour involvement by means of its response to NACT with a tendency to a worse prognosis in these patients.

Nevertheless, the presence of enlarged CPLNs seems to have a rather prognostic value and should therefore not change the current treatment strategy for advanced ovarian cancer. The response to NACT as measured by the CPLN is similar to the response of established parameters like CA125, HE4, ascites, and the Sugarbaker PCI, which gives the CPLN also a monitoring value. Larger studies might be needed to fully evaluate the prognostic role of the CPLN status.

\section{Conflicts of Interest}

The Authors have no conflicts of interest to declare.

\section{Authors' Contributions}

V. Luengas-Wuerzinger, F. Rawert, B. Lampe and P. Mallmann conceived of the presented work. V. Luengas-Wuerzinger. and F. Rawert developed the theory and performed the computations. S. Baransi and S.Classen von Spee verified the analytical methods. V.
Luengas-Wuerzinger wrote the manuscript with support from F. Rawert, K. Carrizo and E. Schuler. P. Mallmann supervised the project. All Authors discussed the results and contributed to the final manuscript.

\section{Acknowledgements}

The Authors thank Mr. Ullrich for the statistical analysis and Dr. med. M. Eiers for the radiological support.

\section{References}

1 Leitlinienprogramm Onkologie: S3-Leitlinie Diagnostik, Therapie und Nachsorge maligner Ovarialtumoren. Version 4.0: 24, March 2020.

2 Reuss A, du Bois A, Harter P, Fotopoulou C, Sehouli J, Aletti G, Guyon F, Greggi S, Mosgaard BJ, Reinthaller A, Hilpert F, Schade-Brittinger C, Chi DS and Mahner S: TRUST: Trial of Radical Upfront Surgical Therapy in advanced ovarian cancer (ENGOT ov33/AGO-OVAR OP7). Int J Gynecol Cancer 29(8): 1327-1331, 2019. PMID: 31420412. DOI: 10.1136/ijgc-2019000682

3 Fagotti A, Ferrandina G, Vizzielli G, Fanfani F, Gallotta V, Chiantera V, Costantini B, Margariti PA, Gueli Alletti S, Cosentino F, Tortorella L and Scambia G: Phase III randomised clinical trial comparing primary surgery versus neoadjuvant chemotherapy in advanced epithelial ovarian cancer with high tumour load (SCORPION trial): Final analysis of peri-operative outcome. Eur J Cancer 59: 22-33, 2016. PMID: 26998845. DOI: 10.1016/j.ejca.2016.01.017

4 Kehoe S, Hook J, Nankivell M, Jayson GC, Kitchener H, Lopes T, Luesley D, Perren T, Bannoo S, Mascarenhas M, Dobbs S, Essapen S, Twigg J, Herod J, McCluggage G, Parmar M and Swart AM: Primary chemotherapy versus primary surgery for newly diagnosed advanced ovarian cancer (CHORUS): an open-label, randomised, controlled, non-inferiority trial. Lancet 386(9990): 249-257, 2015. PMID: 26002111. DOI: 10.1016/S0140-6736(14)62223-6

5 Vergote I, Tropé CG, Amant F, Kristensen GB, Ehlen T, Johnson $\mathrm{N}$, Verheijen RH, van der Burg ME, Lacave AJ, Panici PB, Kenter GG, Casado A, Mendiola C, Coens C, Verleye L, Stuart GC, Pecorelli S, Reed NS, European Organization for Research and Treatment of Cancer-Gynaecological Cancer Group and NCIC Clinical Trials Group: Neoadjuvant chemotherapy or primary surgery in stage IIIC or IV ovarian cancer. N Engl J Med 363(10): 943-953, 2010. PMID: 20818904. DOI: 10.1056/NEJMoa0908806

6 Harter P, Sehouli J, Lorusso D, Reuss A, Vergote I, Marth C, Kim JW, Raspagliesi F, Lampe B, Aletti G, Meier W, Cibula D, Mustea A, Mahner S, Runnebaum IB, Schmalfeldt B, Burges A, Kimmig R, Scambia G, Greggi S, Hilpert F, Hasenburg A, Hillemanns P, Giorda G, von Leffern I, Schade-Brittinger C, Wagner U and du Bois A: A randomized trial of lymphadenectomy in patients with advanced ovarian neoplasms. N Engl J Med 380(9): 822-832, 2019. PMID: 30811909. DOI: 10.1056/NEJMoa1808424

7 Eisenhauer EA, Therasse P, Bogaerts J, Schwartz LH, Sargent D, Ford R, Dancey J, Arbuck S, Gwyther S, Mooney M, Rubinstein L, Shankar L, Dodd L, Kaplan R, Lacombe D and Verweij J: New response evaluation criteria in solid tumours: revised RECIST guideline (version 1.1). Eur J Cancer 45(2): 228-247, 2009. PMID: 19097774. DOI: 10.1016/j.ejca.2008.10.026 
8 Holloway B, Gore M, A'hern R and Parsons C: The significance of paracardiac lymph node enlargement in ovarian cancer. Clinical Radiology 52(9): 692-697, 2019. DOI: 10.1016/S00099260(97)80034-7

9 Prader S, Vollmar N, du Bois A, Heitz F, Schneider S, Ataseven B, Bommert M, Waltering KU, Heikaus S, Koch JA, Alesina PF, Traut A and Harter P: Pattern and impact of metastatic cardiophrenic lymph nodes in advanced epithelial ovarian cancer. Gynecol Oncol 152(1): 76-81, 2019. PMID: 30463683. DOI: 10.1016/j.ygyno.2018.11.001

10 Garbi A, Zanagnolo V, Colombo N, Aletti G, Achilarre MT, Bocciolone L, Landoni F, Rizzo S, Biffi R and Maggioni A: Feasibility of transabdominal cardiophrenic lymphnode dissection in advanced ovarian cancer: initial experience at a tertiary center. Int J Gynecol Cancer 27(6): 1268-1273, 2017. PMID: 28498236. DOI: 10.1097/IGC.0000000000000983

11 Cowan RA, Tseng J, Murthy V, Srivastava R, Long Roche KC, Zivanovic O, Gardner GJ, Chi DS, Park BJ and Sonoda Y: Feasibility, safety and clinical outcomes of cardiophrenic lymph node resection in advanced ovarian cancer. Gynecol Oncol 147(2): 262-266, 2017. PMID: 28888540. DOI: 10.1016/j.ygyno.2017. 09.001

12 Yoo HJ, Lim MC, Song YJ, Jung YS, Kim SH, Yoo CW and Park SY: Transabdominal cardiophrenic lymph node dissection (CPLND) via incised diaphragm replace conventional videoassisted thoracic surgery for cytoreductive surgery in advanced ovarian cancer. Gynecol Oncol 129(2): 341-345, 2013. PMID: 23290988. DOI: 10.1016/j.ygyno.2012.12.023

13 Forstner R, Sala E, Kinkel K, Spencer JA and European Society of Urogenital Radiology: ESUR guidelines: ovarian cancer staging and follow-up. Eur Radiol 20(12): 2773-2780, 2010. PMID: 20839002. DOI: 10.1007/s00330-010-1886-4

14 Luger AK, Steinkohl F, Aigner F, Jaschke W, Marth C, Zeimet AG and Reimer D: Enlarged cardiophrenic lymph nodes predict disease involvement of the upper abdomen and the outcome of primary surgical debulking in advanced ovarian cancer. Acta Obstet Gynecol Scand 99(8): 1092-1099, 2020. PMID: 32112653. DOI: $10.1111 /$ aogs.13835

15 Jacquet P and Sugarbaker PH: Clinical research methodologies in diagnosis and staging of patients with peritoneal carcinomatosis. Cancer Treat Res 82: 359-374, 1996. PMID: 8849962. DOI: 10.1007/978-1-4613-1247-5_23

16 Lampe B, Kroll N, Piso P, Forner DM and Mallmann P: Prognostic significance of Sugarbaker's peritoneal cancer index for the operability of ovarian carcinoma. Int J Gynecol Cancer 25(1): 135144, 2015. PMID: 25423317. DOI: 10.1097/IGC.000000000 0000327

17 Prader S, Harter P, Grimm C, Traut A, Waltering KU, Alesina PF, Heikaus S, Ataseven B, Heitz F, Schneider S and du Bois A: Surgical management of cardiophrenic lymph nodes in patients with advanced ovarian cancer. Gynecol Oncol 141(2): 271-275, 2016. PMID: 26972337. DOI: 10.1016/j.ygyno.2016.03.012

18 Raban O, Peled Y, Krissi H, Goldberg N, Aviram A, Sabah G, Levavi $\mathrm{H}$ and Eitan R: The significance of paracardiac lymphnode enlargement in patients with newly diagnosed stage IIIC ovarian cancer. Gynecol Oncol 138(2): 259-262, 2015. PMID: 26001327. DOI: 10.1016/j.ygyno.2015.05.007
19 Mert I, Kumar A, Sheedy SP, Weaver AL, McGree ME, Kim B and Cliby WA: Clinical significance of enlarged cardiophrenic lymph nodes in advanced ovarian cancer: Implications for survival. Gynecol Oncol 148(1): 68-73, 2018. PMID: 29129390. DOI: 10.1016/j.ygyno.2017.10.024

20 McIntosh LJ, O'Neill AC, Bhanusupriya S, Matalon SA, Van den Abbeele AD, Ramaiya NH and Shinagare AB: Prognostic significance of supradiaphragmatic lymph nodes at initial presentation in patients with stage III high-grade serous ovarian cancer. Abdom Radiol (NY) 42(10): 2513-2520, 2017. PMID: 28429056. DOI: $10.1007 / \mathrm{s} 00261-017-1158-8$

21 Kim TH, Lim MC, Kim SI, Seo SS, Kim SH and Park SY: Preoperative prediction of cardiophrenic lymph node metastasis in advanced ovarian cancer using computed tomography. Ann Surg Oncol 23(4): 1302-1308, 2016. PMID: 26714941. DOI: 10.1245/s10434-015-5015-0

22 Salehi S, Mohammar R, Suzuki C, Joneborg U, Hjerpe E, Torbrand $\mathrm{C}$ and Falconer $\mathrm{H}$ : Cardiophrenic lymph node resection in advanced ovarian cancer: surgical outcomes, pre- and postoperative imaging. Acta Oncol 57(6): 820-824, 2018. PMID: 29182048. DOI: 10.1080/0284186X.2017.1409434

23 Laasik M, Kemppainen J, Auranen A, Hietanen S, Grénman S, Seppänen $\mathrm{M}$ and Hynninen J: Behavior of FDG-avid supradiaphragmatic lymph nodes in PET/CT throughout primary therapy in advanced serous epithelial ovarian cancer: a prospective study. Cancer Imaging 19(1): 27, 2019. PMID: 31142357. DOI: 10.1186/s40644-019-0215-7

24 Lee IO, Lee JY, Kim HJ, Nam EJ, Kim S, Kim SW, Lee CY, Kang WJ and Kim YT: Prognostic significance of supradiaphragmatic lymph node metastasis detected by ${ }^{18} \mathrm{~F}-\mathrm{FDG}$ PET/CT in advanced epithelial ovarian cancer. BMC Cancer 18(1): 1165, 2018. PMID: 30477469. DOI: $10.1186 / \mathrm{s} 12885-018-5067-1$

25 Kolev V, Mironov S, Mironov O, Ishill N, Moskowitz CS, Gardner GJ, Levine DA, Hricak H, Barakat RR and Chi DS: Prognostic significance of supradiaphragmatic lymphadenopathy identified on preoperative computed tomography scan in patients undergoing primary cytoreduction for advanced epithelial ovarian cancer. Int J Gynecol Cancer 20(6): 979-984, 2010. PMID: 20683405. DOI: 10.1111/IGC.0b013e3181e833f5

26 Larish A, Mert I, McGree M, Weaver A, Sheedy S and Cilby W: Recurrence patterns in patients with abnormal cardiophrenic lymph nodes at ovarian cancer diagnosis. Int J Gynecol Cancer 30(4): 504-508, 2020. PMID: 31953350. DOI: 10.1136/ijgc2019-000981
Received August 24, 2021

Revised September 5, 2021

Accepted September 6, 2021 